\title{
Prediction of atmospheric turbulence by means of WRF model for optical communications
}

\begin{abstract}
Alohotsy Rafalimanana, Christophe Giordano, Aziz Ziad, Eric Aristidi
\end{abstract}

Alohotsy Rafalimanana, Christophe Giordano, Aziz Ziad, Eric Aristidi, "Prediction of atmospheric turbulence by means of WRF model for optical communications," Proc. SPIE 11852, International Conference on Space Optics - ICSO 2020, 118524G (11 June 2021); doi: 10.1117/12.2599659

SPIE Event: International Conference on Space Optics - ICSO 2021, 2021, Online Only 


\section{International Conference on Space Optics-ICSO 2020}

Virtual Conference

30 March-2 April 2021

Edited by Bruno Cugny, Zoran Sodnik, and Nikos Karafolas
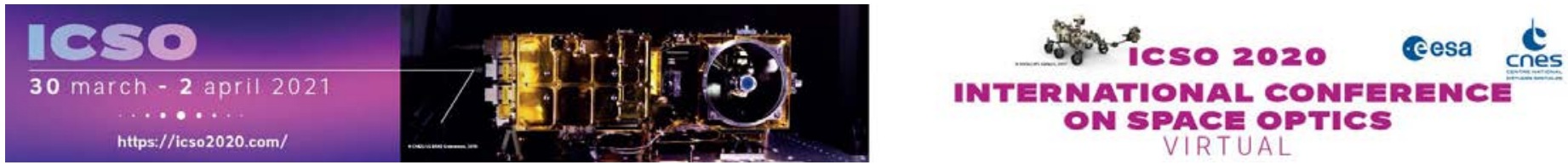

\section{Prediction of atmospheric turbulence by means of WRF model for optical communications}

\section{Cesa isoporecestings denes}




\title{
Prediction of atmospheric turbulence by means of WRF model for optical communications
}

\author{
Alohotsy Rafalimanana, Christophe Giordano, Aziz Ziad, and Eric Aristidi \\ Université Côte d'Azur, Observatoire de la Côte d'Azur, CNRS, J.L. Lagrange, Parc Valrose 06108 \\ Nice Cedex 2, France
}

\begin{abstract}
Free Space Optical (FSO) communication is an excellent tool for high-speed information transmission across a wide range of applications. It is now a key technology to overcome the rapidly increasing bandwidth limitations for space communications. However, FSO is directly affected by atmospheric propagation factors, such as meteorological conditions (cloud, fog, rain, etc.) and atmospheric turbulence conditions. A major challenge among these factors is atmospheric turbulence, which can lead to a phase distortion of the wavefront and to serious degradation of system performance. It is therefore crucial to overcome the effects of atmospheric turbulence in order to reliably monitor, assess the whole performance of the optical system and to fully exploit the potential of the FSO. Forecasts of meteorological and atmospheric turbulence conditions is of interest for optical link optimization because it can contribute to the efficient selection of the optical ground station network. It can also help to identify favorable time periods for laser optical links. In this study, numerical approach by Weather and Research Forecasting (WRF) model coupled with different turbulence models had been used. We compare the vertical profile of meteorological parameters (temperature, relative humidity, wind speed and wind direction) as well as the optical turbulence $\left(C_{n}^{2}\right)$ conditions from the forecast model with the in-situ measurements, balloon-borne radiosoundings launched above Cerro Pachon Observatory in Chile. A new approach to estimate the outer scale $\left(L_{0}\right)$ of turbulence is also presented and discussed.
\end{abstract}

Keywords: Atmospheric turbulence, Free Space Optical Communication, Adaptive optics, Outer scale

\section{INTRODUCTION}

Free Space Optical (FSO) communication is a technology that uses light propagating in free space to transmit information at high bit rates. It offers a good alternative to radio frequency (RF) links which are approaching the limit of their capacities in terms of data rate. FSO communication has attracted more and more attention across a wide range of applications to provide high-speed communication links. These applications include bidirectional ground-satellite laser links, satellite-tosatellite cross-links, up-and-down links between space platforms and aircraft. However, FSO communications are subject to meteorological conditions and atmospheric turbulence that may lead to the degradation of the quality and the reliability of transmissions. Atmospheric turbulence introduces a random variation (in time and space) of the refractive index of the air, causing amplitude phase fluctuations of optical waves. This leads to a considerable degradation of the performance of all systems that use optical waves. ${ }^{1}$ Therefore, weather attenuation losses (such as cloud, fog, rain, haze, smoke and snow) and atmospheric turbulence can be a limiting factor in reliable high-data rate FSO communication link performance. It is therefore important to have a good characterization of the atmosphere in order to reliably monitor, assess the whole performance of the optical system and to fully exploit the potential of the FSO.

Robert et al. (2016) ${ }^{2}$ and Chabé et al. (2020) ${ }^{3}$ have highlighted that FSO communications between earth and satellites is a field in which precise knowledge of the air refractive index structure parameter $\left(C_{n}^{2}\right)$ profiles (as well as wind and outer scale profiles) with high vertical resolution is needed. Thus, the optimization of the performance FSO communications system requires a detailed knowledge of meteorologicals conditions and the optical turbulence (OT) parameters. It is therefore essential to be able to predict, the meteorological parameters (temperature, relative humidity, wind speed and direction) and the profile of $C_{n}^{2}$ because it can contribute to the efficient selection of the optical ground station network and also to specify and optimize the compensation and pre-compensation systems by Adaptive Optics.

Further author information: (Send correspondence to A. Rafalimanana)

Alohotsy Rafalimanana: E-mail: alohotsy.rafalimanana@oca.eu,

Christophe Giordano: E-mail: cgiordano@oca.eu 
In this study, numerical approach by Weather and Research Forecasting ${ }^{4}$ (WRF) model coupled with different turbulence models has been used. Through the use of OT models it is possible to estimate the anticipated range of optical turbulence levels for a particular geographic location and time (i.e. it can help to identify favorable time periods for laser optical links). For the OT models, the outer scale $\left(L_{0}\right)$ of turbulence is an important parameter that need to better take into account. Thus, we have developed a new method based on the Tatarskii model, ${ }^{5}$ to estimate the $L_{0}$.

In this contribution, we performed sets of simulations above the Cerro Pachon Observatory in Chile. For the model validation, we compare the vertical profile of meteorological parameters (temperature, relative humidity, wind speed and wind direction) as well as the optical turbulence conditions (profile $C_{n}^{2}$ ) from the forecast model with the in-situ measurements, balloon-borne radiosoundings launched above Cerro Pachon Observatory in Chile. ${ }^{6}$

In Section 2, we presents the observational data. Section 3 will show the OT model. Section 4 introduces the WRF model setup and simulations. We will present the results of the model validation in Section 5. Section 6 presents the main conclusions.

\section{RADIOSOUNDINGS AND MAST METEOROLOGICAL OBSERVATIONS}

The observational data used in this study came from a measurement campaign during a site testing ${ }^{6}$ that took place at Cerro Tololo and Cerro Pachón in 1998, in the framework of the Gemini program to implement Adaptive Optics (AO) at the focus of their large $8.1 \mathrm{~m}$ telescope. During the campaign, 46 balloons flights measuring the vertical profiles of pressure, temperature, wind speed and direction, relative humidity and $C_{n}^{2}$ were launched from the Cerro Pachón observatory. The radiosoundings have a high vertical resolution $(4-7 \mathrm{~m})$.

The radiosondes were equipped with weather stations to measure meteorological parameters. A pair of sensors (thermal probes) separated by a small distance have been used to measure the temperature structure function. More details about the instrument are explained by Azouit \& Vernin (2005). ${ }^{7}$ The temperature structure function is defined by :

$$
D_{T}(r)=\left\langle[T(x)-T(x+r)]^{2}\right\rangle
$$

where $\mathrm{T}$ is the absolute temperature, \langle\rangle is a temporal average and $r$ is the separation between the two sensors.

In isotropic and homogeneous condition and if the turbulence is within the inertial convective range, the temperature structure function is expressed by the Kolmogorov form:

$$
D_{T}(r)=C_{T}^{2} r^{2 / 3} \quad \text { for } \quad l_{0}<<r<<L_{0}
$$

where $l_{0}$ and $L_{0}$ are the inner and outer scales of the atmospheric turbulence, and $C_{T}^{2}$ is the structure constant of the temperature fluctuations. To derive the $C_{n}^{2}$, we make use of the Gladstone's law:

$$
C_{n}^{2}(z)=\left(\frac{80 \cdot 10^{-6} P(z)}{T(z)^{2}}\right)^{2} \cdot C_{T}^{2}(z)
$$

where $P(z)$ is the vertical profile of atmospheric pressure in $\mathrm{hPa}, T(z)$ is the vertical profile of air temperature in Kelvin

In addition to radiosounding data, we also have in our possession mast meteorological data containing wind profiles for the first 30m (at 3m, 6m, 10m, 15m and 30m) above ground level for 4 days in october 1998. In order to have a mixed profile (mast + radiosounding, in red Fig. 1), an interpolation was made to combine mast data with balloons ones. Fig. 1 shows a vertical profile of wind speed and direction, for the 9 October 1998 where we include data from the mast in the first $30 \mathrm{~m}$ above the surface. It has been found that nacelle balancing affects the quality and reliability of measurements in the first 30-50 meters above the ground. 

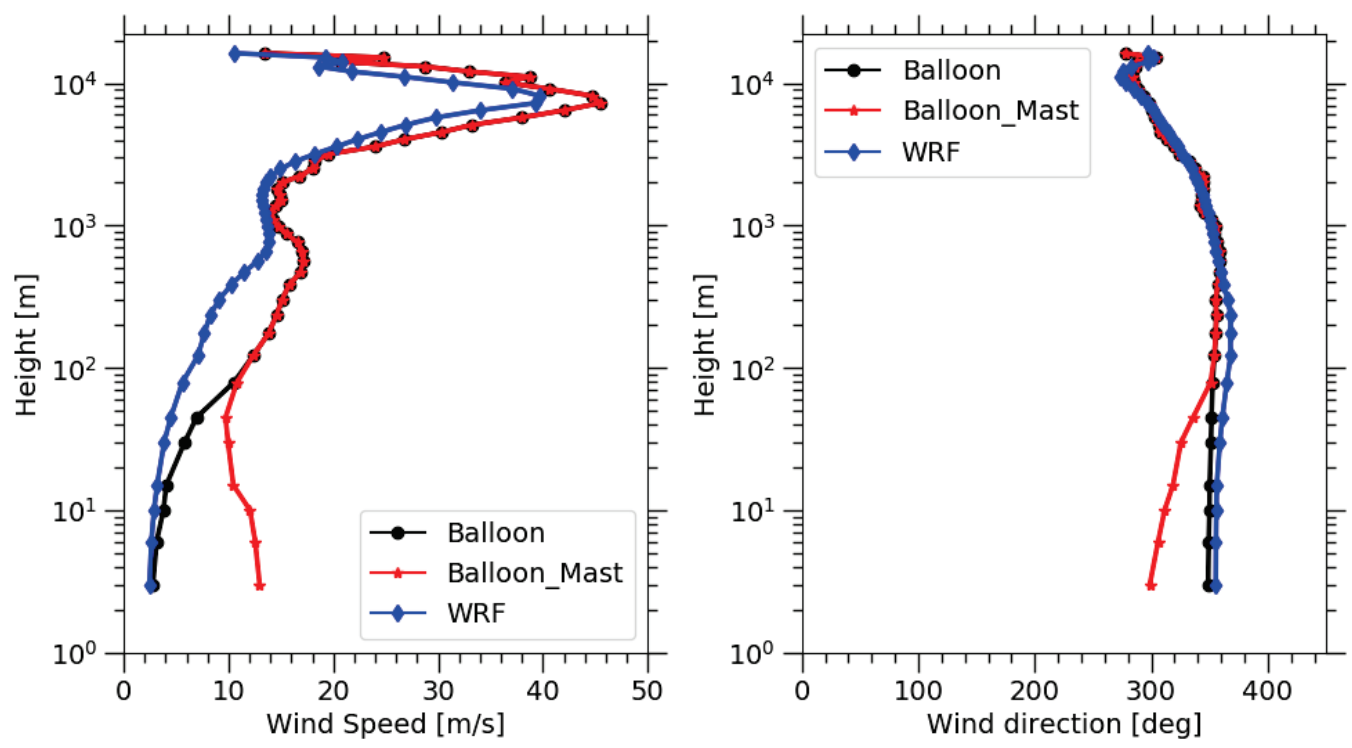

Figure 1: Example of a vertical profile of wind speed and direction, for the 9 October 1998. In black the radiosounding, in blue the prediction by WRF, in red mixed profile (radiosounding + mast).

\section{OPTICAL TURBULENCE MODEL}

\subsection{Model of $C_{n}^{2}$}

The vertical distribution of OT is described by the air refractive index structure constant $C_{n}^{2}$. Tatarskii ${ }^{5}$ in 1961 proposed a theoretical model to compute the $C_{n}^{2}$. In this paper, we called it as WRF_Tatarskii model. $C_{n}^{2}$ is defined by the following equation.

$$
C_{n}^{2}(z)=\gamma M^{2} L_{0}(z)^{4 / 3}
$$

where $\gamma \approx 2.8$, is a constant, $L_{0}$ is the outer scale of the turbulence and $M$ is the vertical gradient of the generalized potential refractive index of the air:

$$
M=-80 \cdot 10^{-6} \frac{P}{g T} N^{2}
$$

where $\mathrm{P}$ is the atmospheric pressure in $\mathrm{hPa}, \mathrm{T}$ is the air temperature in $\mathrm{K}, \mathrm{g}$ is the acceleration due to gravity and $\mathrm{N}$ is the buoyancy frequency given by:

$$
N^{2}=\frac{g}{\theta} \frac{\partial \theta}{\partial z}
$$

where $\theta$ is the potential temperature in $\mathrm{K}$, defined as:

$$
\theta=T\left(\frac{1000}{P}\right)^{R / c_{p}}
$$

where $R=287 \mathrm{JK}^{-1} \mathrm{~kg}^{-1}$ is the gas constant of air and $c_{p}=1004 \mathrm{JK}^{-1} \mathrm{~kg}^{-1}$ is the specific heat capacity at a constant pressure.

\subsection{Outer scale model}

Outer scale $\left(L_{0}\right)$ of turbulence is a relevant parameter for the computation of $C_{n}^{2}$. In this study, we have developed a new method based on the equality between the theoretical model of $C_{n}^{2}$ and the Gladstone's law. The logarithmic differentiation of equation (7) gives:

$$
\frac{1}{\theta} \frac{\partial \theta}{\partial z}=\frac{1}{T} \frac{\partial T}{\partial z}-\frac{R}{C_{p} P} \frac{\partial P}{\partial z}
$$


Considering the hydrostatic equilibrium $\left(\frac{\partial P}{\partial z}=-\rho g\right)$ and the gas law $(P=\rho R T)$, with $\rho$ the density of air, equation (8) becomes:

$$
\frac{1}{\theta} \frac{\partial \theta}{\partial z}=\frac{1}{T} \frac{\partial T}{\partial z}+\frac{1}{T} \frac{g}{C_{p}}
$$

With the dry adiabatic lapse rate $\Gamma_{d}=\frac{g}{C_{p}} \approx 9.8 \times 10^{-3} \mathrm{~K} / \mathrm{m}$, equation (6) can be expressed as a function of the absolute temperature:

$$
N^{2}=\frac{g}{T}\left(\frac{\partial T}{\partial z}+\Gamma_{d}\right)
$$

Using this new expression of $N^{2}$, equation (4) becomes:

$$
C_{n}^{2}(z)=2.8 \cdot\left[-80 \cdot 10^{-6} \frac{P(z)}{T(z)^{2}} \cdot\left(\frac{\partial T}{\partial z}+\Gamma_{d}\right)\right]^{2} \cdot L_{0}(z)^{4 / 3}
$$

Tatarskii ${ }^{5}$ has defined the structure constant of the temperature fluctuations $C_{T}^{2}$ by the following relation

$$
C_{T}^{2}(z)=\alpha L_{0}(z)^{4 / 3}\left(\frac{\partial T}{\partial z}\right)^{2}
$$

Using this expression of $C_{T}^{2}$, the Gladstone's law becomes

$$
C_{n}^{2}(z)=\alpha \cdot\left(80 \cdot 10^{-6} \frac{P(z)}{T(z)^{2}} \cdot \frac{\partial T}{\partial z}\right)^{2} \cdot L_{0}(z)^{4 / 3}
$$

By making equation (11) and (13) equal, we can infer the expression of $\alpha$, given by

$$
\alpha=2.8\left(1+\frac{\Gamma_{d}}{\frac{\partial T}{\partial z}}\right)^{2}
$$

Tatarskii ${ }^{5}$ introduced the mixing scale $\Delta z_{0}$ as the height at which the equality between the temperature systematic difference and the random one is reached. ${ }^{8}$

$$
\left[\langle T(z)\rangle-\left\langle T\left(z+\Delta z_{0}\right)\right\rangle\right]^{2}=\left\langle\left[T(z)-T\left(z+\Delta z_{0}\right)\right]^{2}\right\rangle
$$

The mixing scale $\Delta z_{0}$ is related to the outer scale of turbulence by the relation:

$$
L_{0} \simeq \frac{\Delta z_{0}}{\alpha^{3 / 4}}
$$

A statistical comparison between this new model and the $L_{0}$ deduced directly from the local instantaneous profiles of radiosoundings measurement using equation (4) will be discussed in the Section 5.3.

\section{WRF MODEL SETUP AND SIMULATIONS}

In this study, WRF model was configured with five nested domains of horizontal resolutions varying from $27 \times 27 \mathrm{~km}$ to $0.333 \times 0.333 \mathrm{~km}$ (Fig. 2). The model was run with 46 vertical grid levels with a resolution decreasing slowly with height. The first 16 levels are located in the lowest $1 \mathrm{~km}$ of the atmosphere with the first level at approximately 10 meters above ground level and the model top set at $50 \mathrm{hPa}(\sim 20500 \mathrm{~m}$ above mean sea level). Table 1 shows the main characteristics of the simulation domains. 

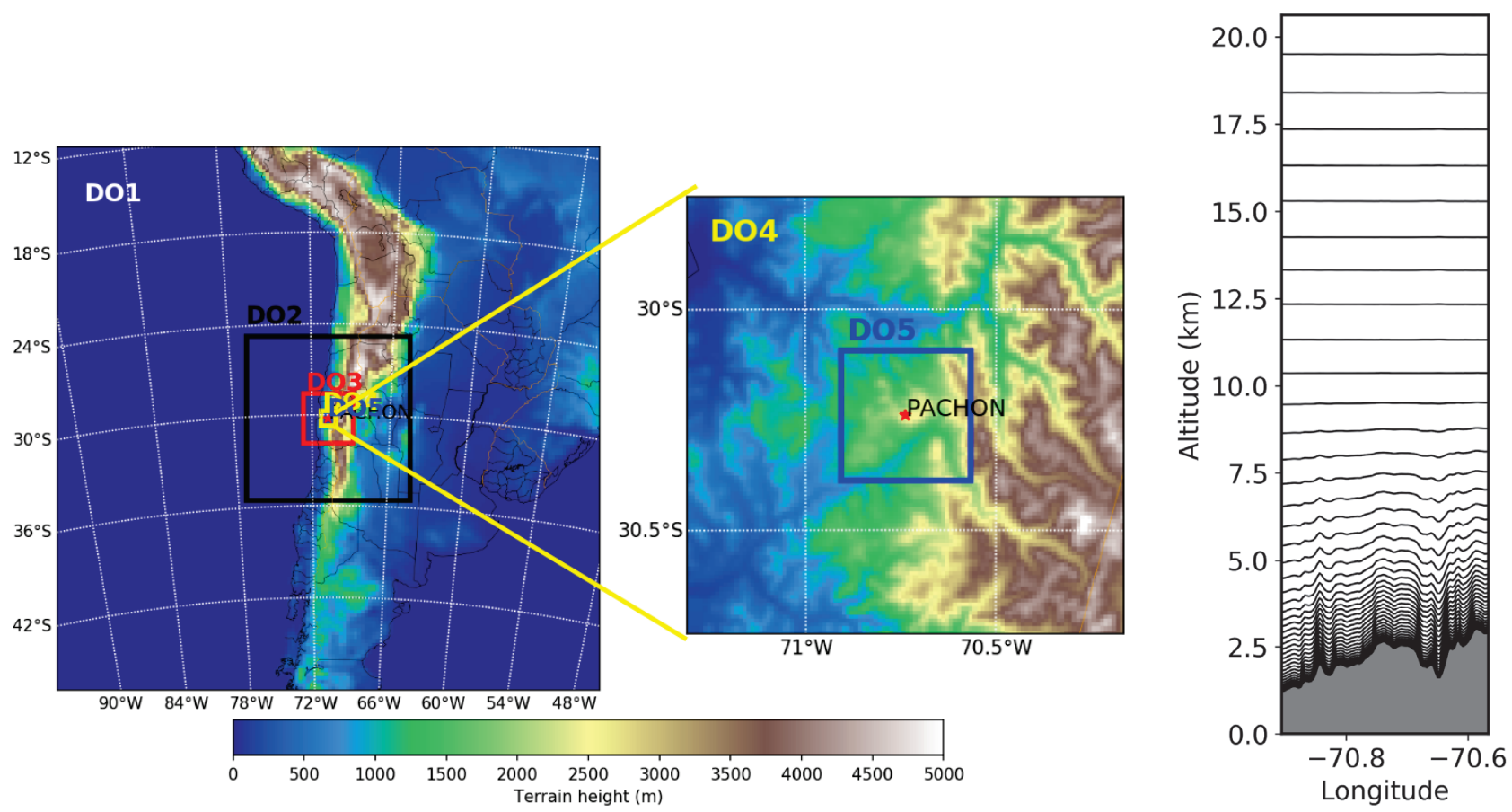

Figure 2: Domains used for our WRF simulations. The horizontal resolution varies from $27 \times 27 \mathrm{~km}$ (D01) to $0.333 \times$ $0.333 \mathrm{~km}$ (D05). Colormap represents the topography. The right plot show the vertical level of the model

Table 1: Simulation domains configuration.

\begin{tabular}{|c|c|c|c|c|}
\hline Domains & Resolution $\left(\mathrm{km}^{2}\right)$ & Grid points number & Vertical level number & Layer thickness $(\mathrm{km})$ \\
\hline D01 & $27 \times 27$ & $150 \times 150$ & & \\
D02 & $9 \times 9$ & $136 \times 136$ & & \\
D03 & $3 \times 3$ & $124 \times 124$ & 46 & $\Delta z_{\min }=0.01$ \\
D04 & $1 \times 1$ & $112 \times 112$ & & $\Delta z_{\text {max }}=1.15$ \\
D05 & $0.333 \times 0.333$ & $100 \times 100$ & & \\
\hline
\end{tabular}

Input data used are those from Climate Forecast System Reanalysis (CFSR) from the National Centers for Environmental Prediction (NCEP). CFSR has a temporal resolution of 6 hours and a horizontal resolution of about $0.5^{\circ} \times 0.5^{\circ}(\sim 56 \times 56$ $\left.\mathrm{km}^{2}\right)$. CFSR data are available at the surface and extend in 64 levels up to $0.266 \mathrm{hPa}(\sim 58500 \mathrm{~m}$ above mean sea level).

The topographic data used come from the Shuttle Radar Topography Mission (SRTM)* having a resolution of $3 \times 3$ $\operatorname{arcsec}(\sim 90 \times 90 \mathrm{~m})$.

Some parameterizations have been used to take into account the physics of the atmosphere. More details about the WRF setup and the physics parameterizations can be found in our previous paper. ${ }^{9}$

Simulations were performed in different periods of the year 1998. For each run, numerical simulations are executed for a 24 hours forecast.

\section{RESULTS}

\subsection{Meteorological parameters}

In this section, WRF model prediction of the vertical profiles of meteorologicals parameters are validated against radiosoundings measurements. The statistical comparison is performed for the whole year 1998.

\footnotetext{
*http://srtm.csi.cgiar.org/
} 
Fig. 3a shows the mean vertical profile of temperature. The mean temperature profiles are very similar over the entire atmosphere. The temperature is the best predicted parameter by WRF model. The colored surface of the standard deviation ovelap over the whole atmosphere.

Fig. 3b shows that WRF model overestimates relative humidity compared to radiosoundings. But regarding the mean value, both measured and predicted profiles are similar. The colored surface of the standard deviation ovelap over the entire atmosphere.

Fig. 3c and Fig. 3d show the mean vertical profiles of wind speed and wind direction with the corresponding standard deviation. Wind speed and wind direction are also well predicted by WRF model in the free atmosphere but still present a difference in the ground layer. Like the temperature and humidity, the colored areas of the standard deviation of the prediction and measurements overlap.

(a)

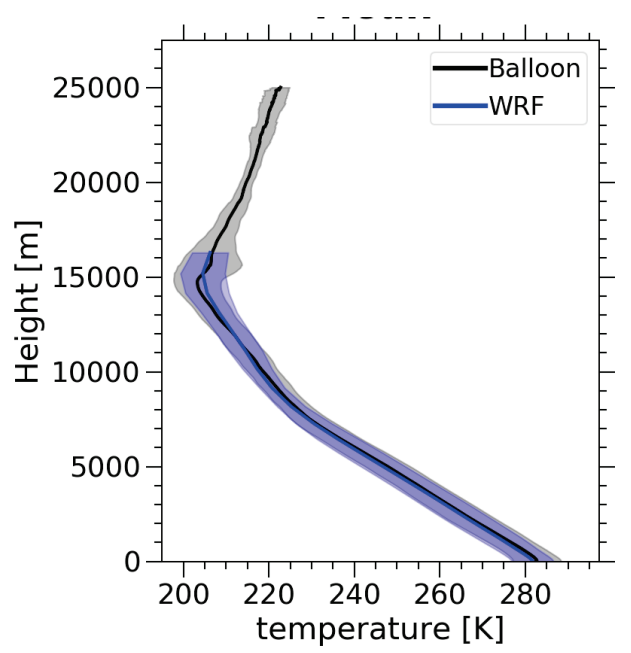

(c)

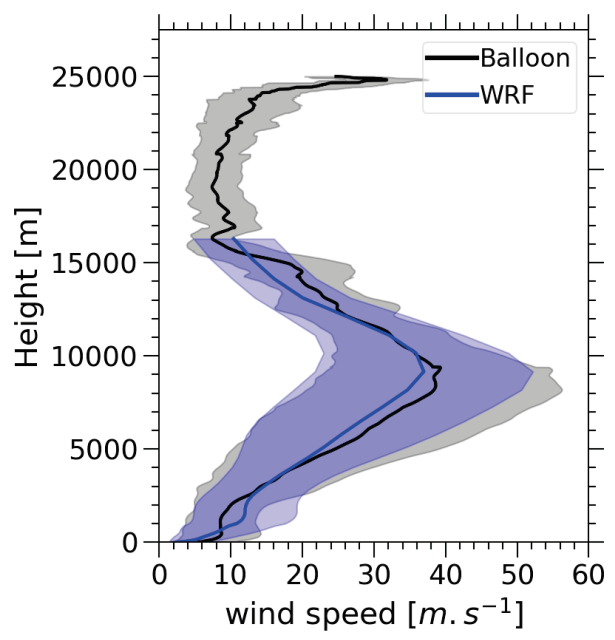

(b)

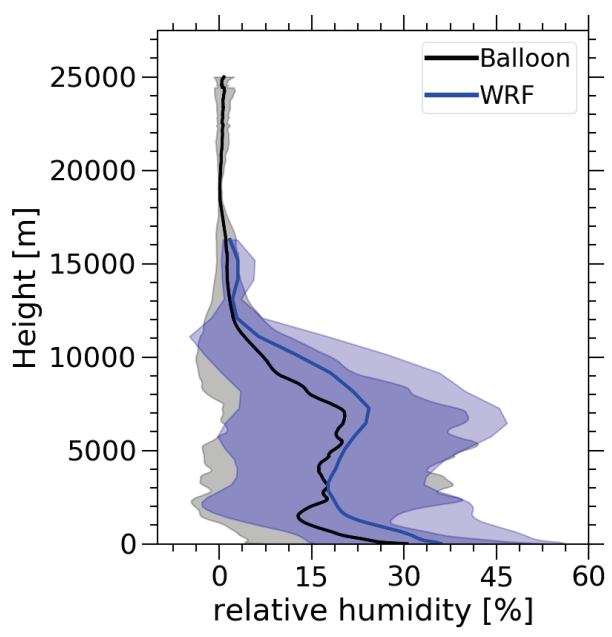

(d)

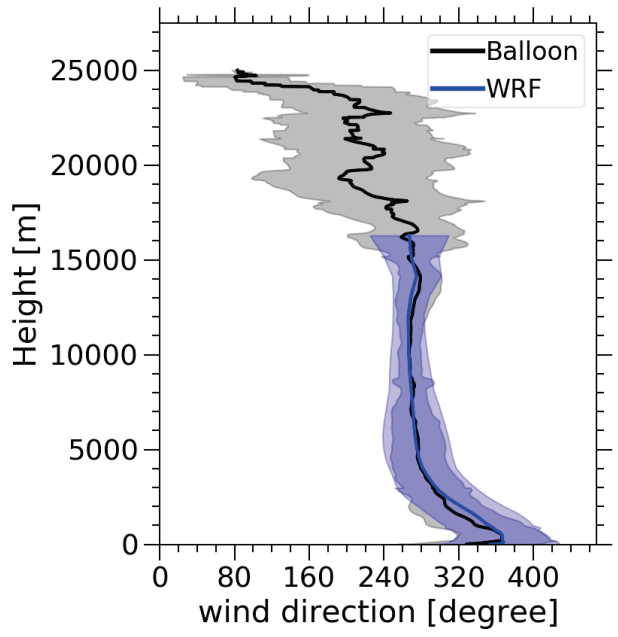

Figure 3: Mean vertical profiles of meteorological parameters for the year 1998. In black line the radiosounding measurements and WRF model in blue line. Temperature (a), relative humidity (b), wind speed modulus (c) and wind direction (d). Colored surface indicate the standard deviation from the mean value 


\subsection{Structure constant of refractive index $C_{n}^{2}$}

The vertical profiles of $C_{n}^{2}$ from the radiosoundings are compared with the estimates from the WRF model. Fig. 4a shows the comparison for the night of 16 April 1998 and Fig. 4b for the night of 11 December 1998. We can see on these two examples that our model of OT is in good agreement with the balloon measurements. The profiles for the cases displayed in Fig. $4 \mathrm{a}$ and Fig. $4 \mathrm{~b}$ are typical of the profile of $C_{n}^{2}$.

(a)

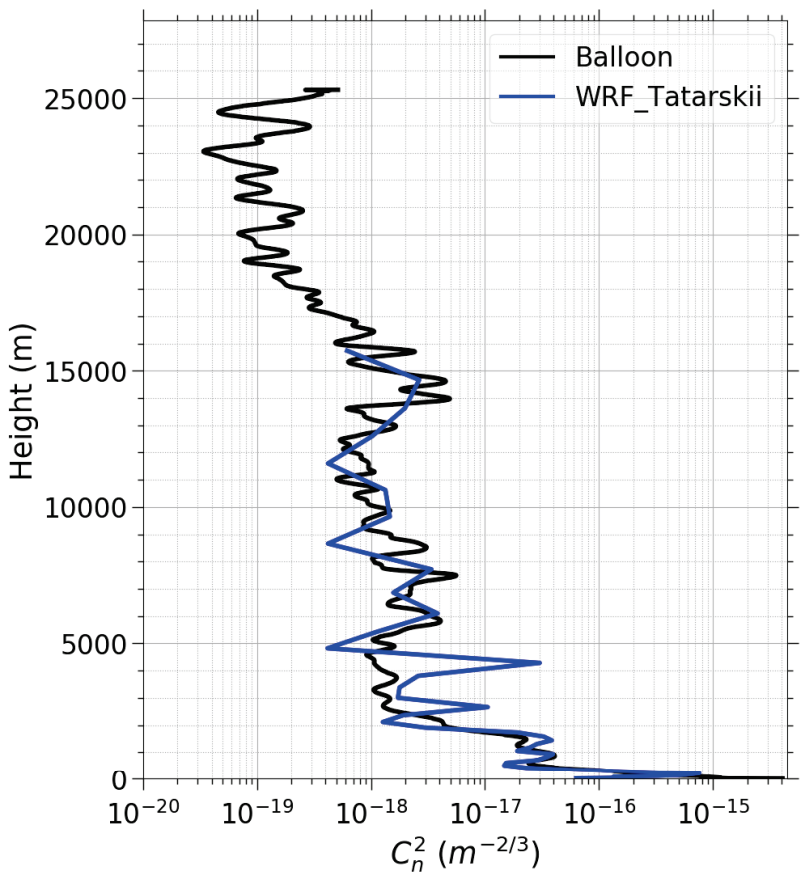

(b)

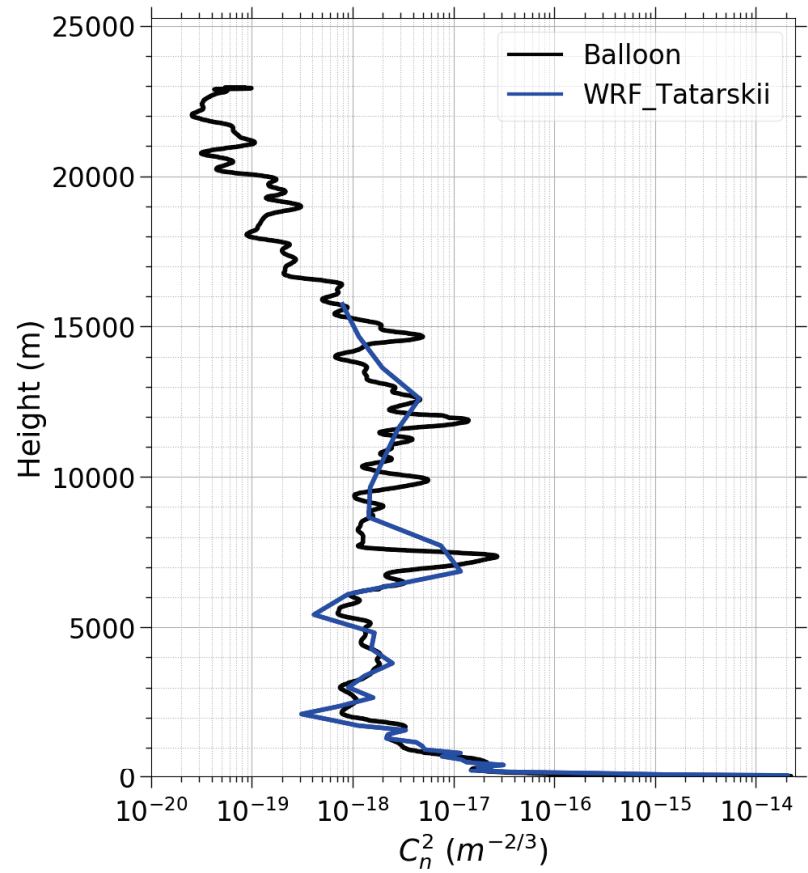

Figure 4: Exemple of a vertical profiles of $C_{n}^{2}$ for the night of the 16 April 1998 (a) and for the night of 11 December 1998 (b). In black line the radiosoundings data and in blue WRF_Tatarskii model

A statistical comparison between the median profile of $C_{n}^{2}$ measured by radiosounding and predicted by WRF with the OT model is shown in Fig. 5. There is generally good agreement between the radiosoundings data and the estimates of $C_{n}^{2}$ from the model. The shape of the profiles is well reconstructed by the OT model compared to the radiosounding profile, except at ground level. Looking at the first and third quartile interval, one can see that colored surfaces are overlapped almost at any altitude. 
(a) Median of $C_{n}^{2}$ for all altitude

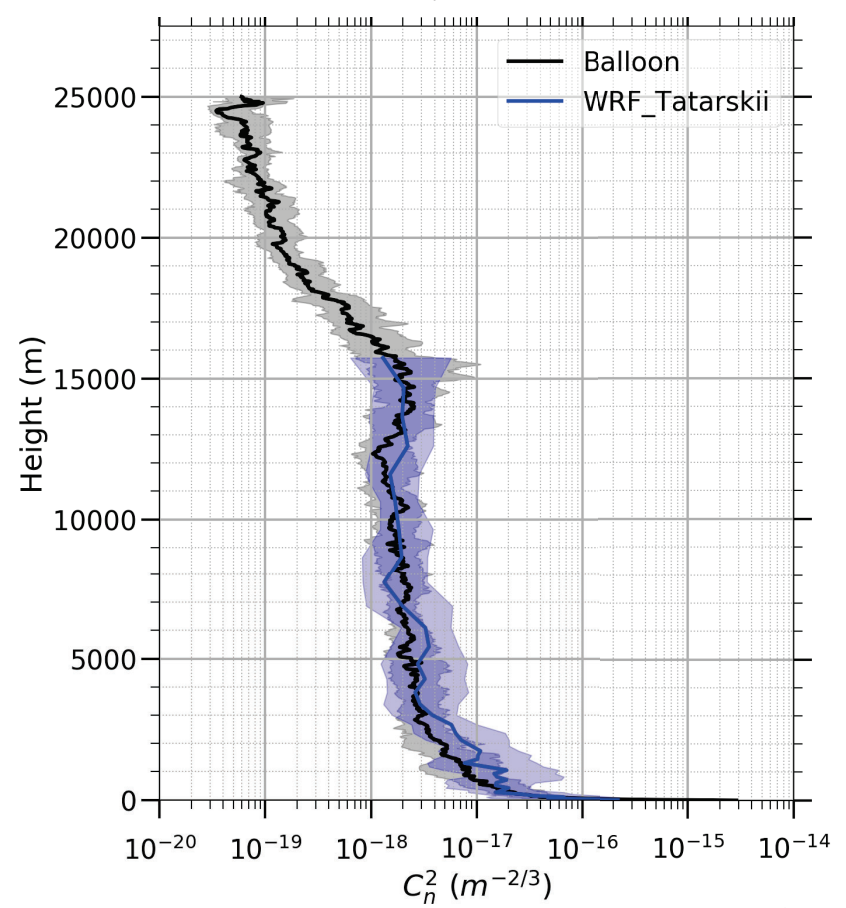

(b) Median of $C_{n}^{2}$ in the PBL

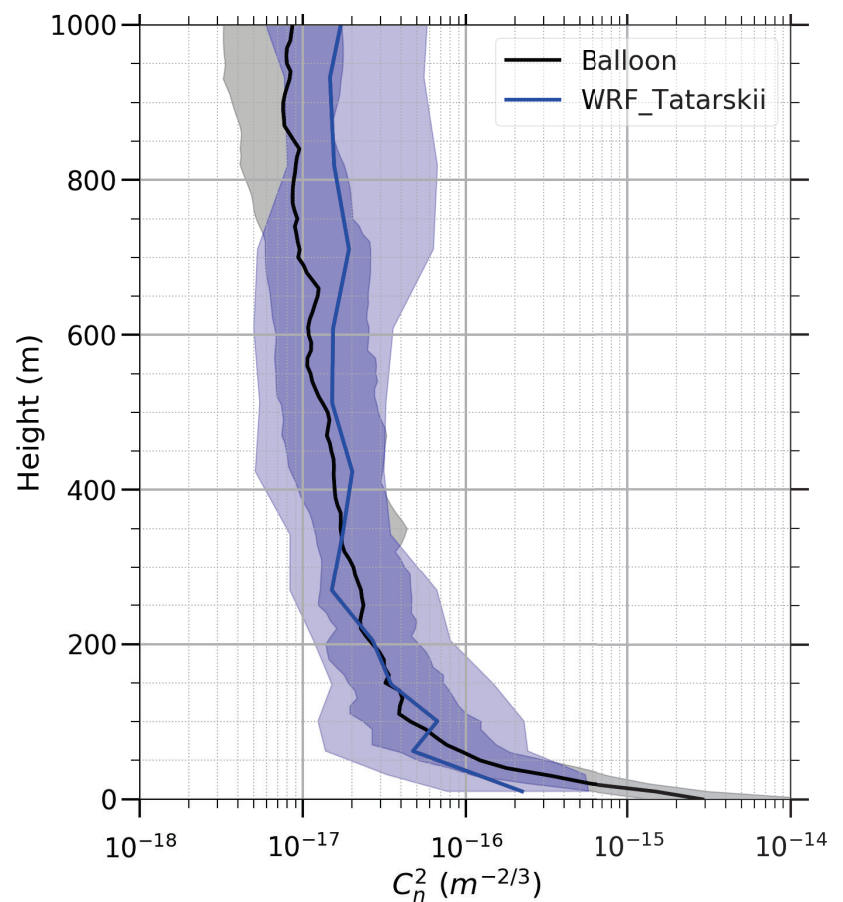

Figure 5: Comparison of the vertical profiles of the $C_{n}^{2}$ deduced from radiosounding (black) and using WRF_Tatarskii model(blue). Thick lines indicate the median value and shading show the interval between the first and the third quartiles. (a) for all altitude and (b) a zoom at the planetary boundary layer.

\subsection{Outer scale of turbulence}

$L_{0}$ used for the calculation of $C_{n}^{2}$ shown in the figures above are deduced directly from the local instantaneous profiles of radiosoundings measurement using equation (4). With these real values of $L_{0}$, the Tatarskii model work well and gives good results. But in the case where direct $L_{0}$ measurements are unavailable, existing $L_{0}$ calculation models can be used to get an estimate. A comparison study between these models and the impact of $L_{0}$ on the prediction of optical turbulence was discussed in a previously submitted paper in MNRAS. ${ }^{10}$ It has been shown that in order to have a good estimate of $C_{n}^{2}$, it is necessary to have a profile of $L_{0}$ as close as possible to the real profile.

We have developed a new approach detailed in section 3.2 to better estimate $L_{0}$. This method needs data with high spatial and temporal resolution. Here we applied it for the data from radiosounding measurement and we compared the result with $L_{0}$ deduced directly from the local instantaneous profiles of radiosoundings using equation (4). The first result of our approch is shown in Fig. 6. Both profiles show almost the same variations with altitude over the whole atmosphere. We can say that there is a correlation between the two profiles, but small difference remain at the height between $1 \mathrm{~km}$ and $6 \mathrm{~km}$. In the boundary layer, our new method of $L_{0}$ is in good agreement with radiosounding balloons data as shown in Fig. 6b. The new method of outer scale extration gives vertical profile of $L_{0}$ that are within the interval between the first and the third quartiles of the balloon data for all altitude. This first step of our study show that the comparison with local measures shows promising agreement. 
(a) $L_{0}$ for all altitude

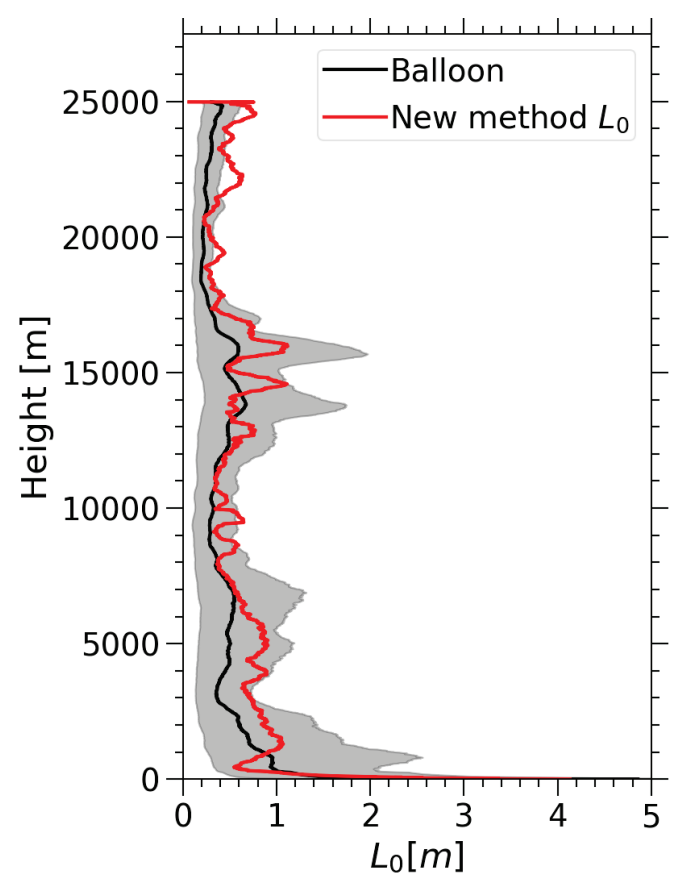

(b) $L_{0}$ in the PBL

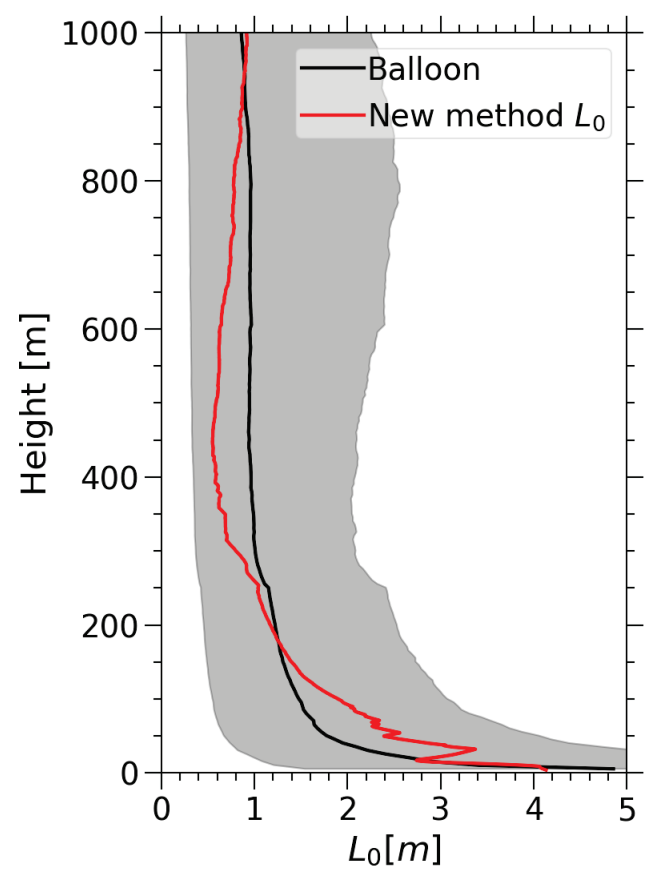

Figure 6: Comparison of the mean vertical profiles of the $L_{0}$ deduced from radiosounding in black and estimated using our new method in red. Grey colored surface shows the interval between the first and the third quartiles of radiosounding data. (a) for all altitude and (b) a zoom at the planetary boundary layer.

\section{CONCLUSION}

In this study, we examined WRF model ability to predict vertical profiles of meteorological parameters and to estimate the OT parameters (vertical profile of $C_{n}^{2}$ ) over the Cerro Pachon Observatory.

We evaluated the differences in meteorological parameters of temperature, relative humidity, wind speed and direction compared with radiosoundings in the innermost domain following a statistical analysis. Based on the evaluation by all available data, we conclude that WRF model produces coherent results of meteorological parameters with radiosounding balloons data.

The vertical profiles of temperature showed an excellent agreement over the entire atmosphere, between WRF and radiosoundings data. WRF overestimates the relative humidity but the standard deviation of the WRF output compared to the observation overlaps for the whole atmosphere. Wind speed and direction are also in a good agreement with the radiosoundings data except in the ground level.

The vertical distributions of OT given by the WRF_Tatarskii model are in good agreement compared to balloons measurements excepted at ground level. This model gives very good estimation of the vertical profile of $C_{n}^{2}$ as long as we have values of $L_{0}$ as exact as possible to the real profile. We developed a new method of outer scale estimation from meteorological data. The preliminary results confirm that this new technique is very promising. More investigations are necessary to make WRF data usable with this new outer scale extraction method.

Our future work intend to continue our studies to improve the estimation of the OT parameters and the outer scale of turbulence by WRF model near the ground. This demands a very good prediction of meteorological parameters that is responsible of generating the OT. Indeed, a project of measurement by instrumented drone on the Calern observatory (France) site is in progress in order to obtain high-resolution vertical profiles of meteorological parameters on the first hundreds of meters above the ground. Having a large data set containing vertical profiles of meteorological parameters within the boundary layer will allow us to better constrain our model of OT. These data will be useful for the evaluation of the prediction by WRF model, and also can be assimilated in WRF as input data in order to improve our prediction. 


\section{ACKNOWLEDGMENTS}

We would like to thank the Centre National d'Études Spatiales (CNES) and the Observatoire de la Côte d'Azur (OCA) for funding this activity. We acknowledge the NCEP/NCAR for the availability of the WRF model and thank the NCEP/NOAA for providing public access to the CFSR data used in this study. We also extend our thanks to SRTM for giving access to the high-resolution topographic data. We are also grateful to the Mesocentre SIGAMM of the Observatoire de la Côte d'Azur for hosting our model and for their help throughout the installation process.

\section{REFERENCES}

[1] Andrews, L. C. and Phillips, R. L., [Laser Beam Propagation through Random Media], SPIE (2005).

[2] Robert, C., Conan, J.-M., and Wolf, P., "Impact of turbulence on high-precision ground-satellite frequency transfer with two-way coherent optical links," Phys. Rev. A 93, 033860 (Mar 2016).

[3] Chabé, J., Aristidi, E., Ziad, A., Lantéri, H., Fanteï-Caujolle, Y., Giordano, C., Borgnino, J., Marjani, M., and Renaud, C., "Pml: a generalized monitor of atmospheric turbulence profile with high vertical resolution," Appl. Opt. 59, 7574-7584 (Sep 2020).

[4] Skamarock, W. C., Klemp, J. B., Dudhia, J., Gill, D. ., Liu, Z., Berner, J., Wang, W., Powers, J. G., Duda, M. G., Barker, D. M., and Huang, X.-Y., "A Description of the Advanced Research WRF Version 4," NCAR Tech. Note NCAR/TN-556+STR, 145pp. (2019).

[5] Tatarskii V., I. and Silverman R., A., [Wave Propagation in a Turbulent Medium], Dover books on physics and mathematical physics, Dover (1961).

[6] Vernin, J., Agabi, A., Avila, R., Azouit, M., Conan, R., Martin, F., Masciadri, E., Sanchez, L., and Ziad, A., "1998 gemini site testing campaign: Cerro pachon and cerro tololo," tech. rep. (2000).

[7] Azouit, M. and Vernin, J., "Optical turbulence profiling with balloons relevant to astronomy and atmospheric physics," PASP 117, 536-543 (may 2005).

[8] Ziad, A., "Review of the outer scale of the atmospheric turbulence," in [Adaptive Optics Systems V], Marchetti, E., Close, L. M., and Véran, J.-P., eds., 9909, 545 - 556, International Society for Optics and Photonics, SPIE (2016).

[9] Rafalimanana, A., Giordano, C., Ziad, A., and Aristidi, E., "Toward an optimal prediction of atmospheric turbulence by means of WRF model," in [Adaptive Optics Systems VII], 11448, 1490 - 1500, SPIE (2020).

[10] Rafalimanana, A., Giordano, C., Ziad, A., and Aristidi, E., "Optimal prediction of atmospheric turbulence by means of WRF model," submitted (2021). 